\title{
El último consumo de alcohol previo al ingreso de desintoxicación como predictor a largo plazo de la abstinencia.
}

\author{
Miouel Monrás*, Anna Lligoña**, Neus FreiXa*, Antoni Gual*** \\ Unidad de Alcohologia, Institut Clínic de Psiquiatria i Psicología. (ICPP) Hospital Clínic de Barcelona. \\ * Psicólogo Adjunto, ** Médico Psiquiatra Adjunto, ** Jefe de Sección. \\ Enviar correspondencia a: \\ Miquel Monras. Unidad de Alcohología. Recinto Hospital Casa Maternidad (Hospital Clínic de Barcelona). C/ Mejía Lequerica 1. Edificio Helios II. \\ 08028 BarcelonaTeléfono: (+34) 93.227.99.23. Fax: (+34) 93.227.56.88. e-mail: mmonras@clinic.ub.es.
}

\section{RESUMEN}

Se estudia la utilidad del fenómeno de la "despedida" del consumo de alcohol como indicador de resistencia al cambio de fase del estado motivacional de los pacientes alcohólicos y si puede predecir la evolución terapéutica posterior. A una cohorte de 71 alcohólicos ingresados consecutivamente para desintoxicación enólica se les realiza alcoholuria inmediatamente después del ingreso y son evaluados al cabo de un año. La alcoholuria resultó positiva en el $47,9 \%$ de pacientes. Este porcentaje fue similar para ambos sexos e independiente de la existencia de antecedentes de tratamiento o consumo de otras drogas. Al año no se hallan diferencias en la abstinencia o el cumplimiento de tratamientos entre pacientes con alcoholuria positiva o negativa. Sin embargo en los que han hecho tratamientos previos la alcoholuria positiva es un elemento que empeora el pronóstico. Se concluye que la ausencia de "despedida" no es buen indicador de un cambio en la motivación ni del pronóstico terapéutico si no se tienen en cuenta a otros factores.

Palabras clave: Alcoholismo, análisis toxicológico, pronóstico, motivación, estados del cambio.

\section{ABSTRACT}

We studied the utility of the "one last drink" prior to entering an inpatient detoxification programme as an indicator of resistance to a change of phase in the motivational state of alcoholic patients and if it could predict posterior therapeutic evolution. Urine samples of a cohort of 71 consecutive admissions for alcohol inpatient detoxification were obtained immediately after admission, and the results were compared with baseline and one year follow-up data. $47.9 \%$ of the samples screened positive for alcohol. This percentage was similar for both sexes irrespective of the existence of earlier treatment or the use of other drugs. No differences were found in abstinence or in compliance with treatment among patients who tested positive or negative for alcohol at one-year follow-up. However, in those who had undergone previous treatment, a positive analysis was a factor that predicted a poorer clinical evolution. It is concluded that the absence of "one last drink" is not a good indicator of a change in motivation or of therapeutic prognosis if other factors are not taken into account.

Key words: Alcoholism, detoxification, prognosis, motivation.

\section{INTRODUCCIÓN}

E enfoque motivacional en el tratamiento de las drogodependencias ha ido adquiriendo de forma paulatina una mayor relevancia en los últimos años ?

La teoría transteórica del cambio ${ }^{2,3}$ describe como los individuos evolucionan por distintas fases antes de superar sus problemas de adicción. Este proceso evolutivo se llama rueda del cambio y se compone de seis etapas por las que pasa el paciente tanto si recibe ayuda de un terapeuta como si no: I) pre-contemplativa, en que hay una ausencia de intención de cambiar en un futuro próximo, II) contemplativa, caracterizada por la ambivalencia, III) preparación para la acción, en que está decidido y ya se plantea de forma próxima los medios y estrategias para cambiar, IV) acción, en que pone en marcha los medios de forma concreta, $V$ ) mantenimiento del cambio realizado y VI) recaída, en que reinicia el proceso en la fase de contemplación. 
Desde la perspectiva de la rueda del cambio es importante conocer cómo un alcohólico va evolucionando a través de sus distintas fases, hasta que llega al punto de eliminar su consumo de tóxicos y posteriormente mantener la abstinencia.

Diversas verbalizaciones y autoafirmaciones de los pacientes pueden servirnos para situar en que fase del cambio se encuentran y con ello saber que estrategias debe utilizar el clínico. También algunas conductas pueden ser indicativas del inicio de un cambio en la actitud del enfermo ante su consumo de tóxicos.

En el caso de los alcohólicos severos, que requieren un ingreso para su desintoxicación, este ingreso representa una ruptura, que aunque legalmente voluntaria acostumbra a ser impuesta por la gravedad de las consecuencias que ha ocasionado el alcohol. Ello ocasiona una resistencia a su abandono, que en definitiva es el reflejo de la lucha interna provocada por una dependencia a substancias, tanto en su vertiente psíquica como física.

Ante el ingreso, aunque también y en menor medida ante las desintoxicaciones ambulatorias, se produce frecuentemente el fenómeno de la "despedida" que se caracteriza porque el enfermo alcohólico realiza un último consumo de esta sustancia, a menudo puntual y muy abusivo, de forma inmediatamente anterior al ingreso.

En el contexto del proceso de cambio los terapeutas suponemos que esta conducta de despedida es un indicador de resistencia ante el cambio, que viene impuesto desde el exterior. Por el contrario los enfermos que intentan disminuir o incluso eliminar sus consumos con anterioridad al ingreso, serian personas que ya han decidido por su cuenta iniciar un proceso de abstinencia.

Una hipótesis resultante de la anterior es suponer que el pronóstico a medio plazo de unos y otros debería ser distinto, por cuanto se encontrarían en fases distintas de la rueda del cambio motivacional.

El objetivo de este trabajo es comprobar estas hipótesis, investigando la posible relación entre el consumo de alcohol inmediato al ingreso de desintoxicación (la "despedida"), el mantenimiento posterior de la abstinencia y el cumplimiento del tratamiento al cabo de un año.

Los autoinformes del paciente respecto de sus consumos recientes a menudo son muy poco fiables, y la información de colaterales es poco útil, sobretodo si los patrones de consumo son a escondidas, irregulares o suceden en momentos en que no existe ningún control externo, como los fines de semana. En estos casos la determinación de alcohol en orina es la mejor técnica para la detección de los consumos recientes ${ }^{4}$. Es importante señalar que otros marcadores más clásicos, como GGT, GOT/GOT o CDT valoran consumos regulares en los últimos días, pero no responden al objetivo de medir un cambio muy reciente de la conducta ${ }^{5}$, ante un acontecimiento como puede ser el ingreso hospitalario.

Así, si entendemos la alcoholuria como una forma de monitorización de la abstinencia y la abstinencia como una medida de la motivación al tratamiento, el objetivo del presente estudio es conocer si podemos considerar que la alcoholuria de ingreso puede servir como factor pronóstico de la evolución de la abstinencia al año de tratamiento.

\section{MATERIAL Y MÉTODO:}

Estudio de seguimiento al año de una cohorte de 71 pacientes alcohólicos ingresados consecutivamente desde Enero a Junio de 1998 en la Unidad de Desintoxicación Hospitalaria de la Unidad de Alcohologia del Hospital Clínico de Barcelona (UA-HCB) para la realización de una desintoxicación alcohólica.

Criterios de inclusión: dependencia al alcohol según criterios DSM-IV. Ingreso para desintoxicación alcohólica.

Criterios de exclusión: Pacientes que ingresan procedentes de otros centros de tratamiento de drogodependencias (CAD) y que al alta siguen de nuevo el tratamiento ambulatorio en ellos.

Se admiten pacientes que presenten dependencia concomitante a otras substancias.

\subsection{Procedimiento:}

Todos los pacientes siguen el protocolo de desintoxicación física alcohólica mediante medicación tranquilizante con tolerancia cruzada con el alcohol, y se inicia el proceso de deshabituación psicológica en que se utilizan tanto fármacos anticraving o antidipsotrópicos, como visitas médicas y psicológicas diarias, terapia grupal en días alternos, una sesión de video-forum y una sesión informativa en grupo, un grupo para los familiares y controles toxicológicos diarios. La duración media del ingreso es de unos 7 días.

A continuación del alta hospitalaria los pacientes siguen asistiendo por espacio de 2 semanas a las sesiones grupales en días alternos y acudiendo al mismo tiempo a controles toxicológicos en orina. Posteriormente siguen tratamiento ambulatorio por un periodo de 2 años, consistente en visitas psiquiátricas, psicológicas y con un médico internista, así como terapia grupal en los casos candidatos a ella.

En el día de ingreso se hace una evaluación médica general, un historial toxicológico con vistas a programar el tratamiento de desintoxicación adecuado y 
una analítica general en suero, que incluye bioquímica, hematología y microbiología hepática. Así mismo se realiza un screening toxicológico en orina. La recogida de las muestras se efectúa durante la hora posterior al ingreso hospitalario, que se realiza siempre por la mañana.

Dentro de este análisis de ingreso se incluye la evaluación del consumo reciente de alcohol mediante determinación cuantitativa de etanol en orina (alcoholuria) en mg/l mediante la técnica enzimática del alcohol deshidrogenasa (Roche-Diagnostic $®$ ). Posteriormente en la mayoría de análisis estadísticos se ha transformado este valor a cualitativo dicotómico (positivo/negativo).

Todos los pacientes han sido reevaluados de su consumo de alcohol y revisado su cumplimiento del tratamiento al año del alta hospitalaria.

\subsection{Análisis estadístico:}

Frecuencias, medias y desviaciones típicas para la descripción de los sujetos. T de Student para la comparación de medias entre los pacientes abstinentes (alcoholuria de ingreso negativa) y los consumidores (alcoholuria positiva) y Chi al cuadrado $\left(\chi^{2}\right)$ para comparaciones con variables cualitativas.

Correlación de Pearson entre el valor cuantitativo de la alcoholuria en el momento del ingreso y las demás variables cuantitativas (GOT, GPT, etc.)

\section{RESULTADOS:}

\section{1. Datos del ingreso:}

El $63 \%$ de la muestra son hombres y la edad media

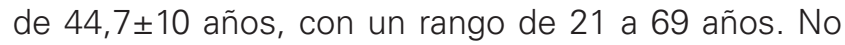
hay diferencias de edad entre ambos sexos.

$47,9 \%$ de pacientes han dado positivo en su alcoholúria de ingreso. La Tabla 1 muestra la media y el rango entre los positivos.

No existe diferencia significativa en el porcentaje de positivos entre ambos sexos. Tampoco si se considera la alcoholúria cuantitativamente, tanto si tenemos en cuenta a todos los pacientes como sólo a los que el resultado fue positivo.

El valor medio en la analítica general del día de ingreso de las GOT y GPT es de $54 \pm 50$ y $56 \pm 90$ respectivamente. El $45 \%$ de pacientes ya habían realizado tratamientos anteriores por su alcoholismo y el $31 \%$ consumían otras drogas. La Tabla 1 muestra como no se han encontrado diferencias de estos valores en función del resultado de la alcoholúria de entrada. Tampoco existe correlación de las GOT y GPT con la alcoholúria cuantitativa entre los pacientes que la han tenido positiva.

\subsection{Datos al año:}

La Tabla 1 muestra para los pacientes con alcoholuria positiva o negativa la falta de diferencias en los porcentajes de los que siguen en tratamiento, han

Tabla 1. Diferencias entre los pacientes con alcoholúria de ingreso positiva y negativa.

\begin{tabular}{|c|c|c|c|}
\hline & $\begin{array}{l}\text { Alcoholúria } \\
\text { positiva } \\
N=34\end{array}$ & $\begin{array}{l}\text { Alcoholúria } \\
\text { negativa } \\
\mathrm{N}=37\end{array}$ & $\begin{array}{c}\text { Prueba y } \\
\text { significación } \\
\text { estadística }\end{array}$ \\
\hline \multicolumn{4}{|l|}{ Datos al ingreso } \\
\hline Alcoholúria media & $930 \pm 838 \mathrm{mg} / \mathrm{l}$ & 0 & \\
\hline Rango de la alcoholúria positiva & 79 a 2696 mg/l & 0 & \\
\hline Edad & $44,9 \pm 8$ & $44,6 \pm 11$ & $t=0,12 p=$ n.s. \\
\hline GOT & $60,7 \pm 42$ & $48,7 \pm 55$ & $t=1 p=n . s$ \\
\hline GPT & $73,8 \pm 122$ & $41,7 \pm 43$ & $t=1,4 p=$ n.s. \\
\hline Hombres & $61,8 \%$ & $64,9 \%$ & $\mathrm{Ji}^{2}=0,07 \mathrm{p}=$ n.s. \\
\hline Tratamientos alcohológicos previos & $47 \%$ & $43 \%$ & $J i^{2}=0,1 p=$ n.s. \\
\hline Consumo de otras drogas & $20,6 \%$ & $40,5 \%$ & $\mathrm{Ji}^{2}=3,1 \mathrm{p}=$ n.s. \\
\hline \multicolumn{4}{|l|}{ Situación al año } \\
\hline Sigue en tratamiento & $44 \%$ & $51 \%$ & $J i^{2}=0,3 p=$ n.s. \\
\hline No abstinentes o abandono de tratamiento. & $67 \%$ & $59 \%$ & $J i^{2}=0,5 p=$ n.s. \\
\hline Indicación de terapia grupal & $17,6 \%$ & $21,6 \%$ & $\mathrm{Ji}^{2}=0,1 \mathrm{p}=$ n.s. \\
\hline Indicación de alcoholurias & $88 \%$ & $78 \%$ & $\mathrm{Ji}^{2}=1,2 \mathrm{p}=$ n.s. \\
\hline Acuden a alcoholurias (de los indicados) & $26,7 \%$ & $44,8 \%$ & $\mathrm{Ji}^{2}=2,1 \mathrm{p}=$ n.s. \\
\hline
\end{tabular}


abandonado o consumido alcohol, se les ha indicado terapia grupal (indicador indirecto de buen cumplimiento) y de los que se les ha indicado y han acudido a controles toxicológicos de orina seriados realizados de forma ambulatoria.

Se ha analizado también si el consumo de otras drogas podía ser una variable que tuviera mejor utilidad pronóstica al año (Tabla 2). Tanto respecto a los porcentajes de seguimiento del tratamiento, de abstinencia, de indicaciones de terapia grupal, de indicaciones o de cumplimiento de los controles toxicológicos en orina el resultado ha sido la falta de significación estadística en estas relaciones. En cambio los antecedentes de tratamientos alcohológicos han mostrado una fuerte relación con unos peores porcentajes de abstinencia y una menor frecuencia de indicaciones de terapia grupal al año. Esto último seguramente se relaciona con la inefectividad de las reindicaciones grupales ${ }^{8}$, lo que lleva a los profesionales de sala a no malgastarlas en los pacientes que ya han tenido diversas experiencias grupales durante sus estancias hospitalarias.

En vista de estos resultados posteriormente hemos comparado la alcoholuria de ingreso con las variables de seguimiento al año, pero estratificada según hubieran antecedentes de tratamiento alcohológico o no.
El resultado de nuevo ha sido el de no encontrar significación estadística en las diferencias.

A pesar de ello se observa una tendencia a que las relaciones estadísticas entre la alcoholuria y la situación al año sean mas intensas en los pacientes que han tenido tratamientos previos. Por ello se ha generado una nueva variable combinación del resultado de la alcoholuria de ingreso y de los antecedentes de tratamiento (Tabla 3): el único resultado significativo es que se indica más frecuentemente la terapia grupal a los pacientes sin antecedentes de tratamientos alcohológicos previos, independientemente del resultado de la alcoholúria de ingreso. En la tabla 3 también se observa que los pacientes con antecedentes de tratamientos alcohológicos previos y además alcoholúria positiva son los de peor pronóstico.

\section{DISCUSIÓN:}

Los datos indican la falta de relación directa entre el consumo de alcohol previo al ingreso de desintoxicación (la "despedida") con la situación clínica y toxicológica de los pacientes al cabo de un año.

Tabla 2. Diferencias al año entre los pacientes con o sin antecedentes de tratamientos alcohológicos.

\begin{tabular}{|l|c|c|c|}
\hline Situación al año & $\begin{array}{c}\text { Con antecedentes } \\
\text { de tratamientos } \\
\text { alcohológicos previos } \\
\mathbf{N = 3 2}\end{array}$ & $\begin{array}{c}\text { Sin antecedentes } \\
\text { de tratamientos } \\
\text { alcohológico previos } \\
\mathbf{N = 3 9}\end{array}$ & $\begin{array}{c}\text { Prueba y } \\
\text { significación } \\
\text { estadística }\end{array}$ \\
\hline Sigue en tratamiento & $40,6 \%$ & $53,8 \%$ & $\mathrm{Ji}^{2}=1,2 \mathrm{p}=$ n.s. \\
\hline No abstinentes o abandono de tratamiento. & $78 \%$ & $51 \%$ & $\mathrm{Ji}^{2}=5,4 \mathrm{p}=0,01$ \\
\hline Indicación de terapia grupal & $3 \% 33 \%$ & $\mathrm{Ji}^{2}=10,1 \mathrm{p}=0,001$ & \\
\hline Indicación de alcoholurias & $90,6 \%$ & $76,9 \%$ & $\mathrm{Ji}^{2}=2,3 \mathrm{p}=$ n.s. \\
\hline Acuden a alcoholurias (de los indicados) & $34,5 \%$ & $36,7 \%$ & $\mathrm{Ji}^{2}=0,03 \mathrm{p}=$ n.s. \\
\hline
\end{tabular}

Tabla 3. Relación entre la alcoholuria de ingreso combinada con los antecedentes de tratamientos alcohológicos previos y las variables clínicas al año del alta hospitalaria.

\begin{tabular}{|l|c|c|c|c|c|}
\hline Situación al año & $\begin{array}{c}\text { OH+TRAC+ } \\
\mathbf{N = 1 6}\end{array}$ & $\begin{array}{c}\text { OH+TRAC- } \\
\mathbf{N = 1 8}\end{array}$ & $\begin{array}{c}\text { OH-TRAC+ } \\
\mathbf{N = 1 6}\end{array}$ & $\begin{array}{c}\text { OH-TRAC- } \\
\mathbf{N = 2 1}\end{array}$ & $\begin{array}{c}\text { Prueba y significación } \\
\text { estadística }\end{array}$ \\
\hline Sigue en tratamiento & $31,3 \%$ & $55,6 \%$ & $50 \%$ & $52,4 \%$ & $\mathrm{Ji}^{2}=2,3 ; \mathrm{p}=\mathrm{n} . \mathrm{s}$. \\
\hline $\begin{array}{l}\text { No abstinentes o abandono } \\
\text { de tratamiento. }\end{array}$ & $87,5 \%$ & $50 \%$ & $68,8 \%$ & $52,4 \%$ & $\mathrm{Ji}^{2}=6,6 ; \mathrm{p}=$ n.s. \\
\hline Indicación de terapia grupal & $0 \%$ & $33,3 \%$ & $6,3 \%$ & $33,3 \%$ & $\mathrm{Ji}^{2}=10,3 ; \mathrm{p}=0,01$ \\
\hline Indicación de alcoholurias & $0 \%$ & $22,2 \%$ & $18,8 \%$ & $23,8 \%$ & $\mathrm{Ji}^{2}=4,3 ; \mathrm{p}=$ n.s. \\
\hline $\begin{array}{l}\text { Acuden a alcoholurias } \\
\text { (de los indicados) }\end{array}$ & $18,8 \%$ & $35,7 \%$ & $53,8 \%$ & $37,5 \%$ & $\mathrm{Ji}^{2}=3,8 ; \mathrm{p}=$ n.s. \\
\hline
\end{tabular}

$\mathrm{OH}$ : Alcoholuria de ingreso; $\mathrm{OH}+$ : positiva; $\mathrm{OH}-:$ negativa;

TRAC: Antecedentes de tratamientos alcohológicos previos TRAC+: Con antecedentes; TRAC-: Sin antecedentes. 
Esta relación a los 5 años sí que se ha demostrado que existe en el caso de pacientes alcohólicos tratados ambulatoriamente ${ }^{9}$.

- Una primera explicación es que el fenómeno de la despedida, medida por la alcoholuria de ingreso, simplemente no es un buen indicador del estadio del cambio y tampoco de la evolución posterior.

- Probablemente esto no es del todo cierto y la explicación es mucho más compleja, debiendo aglutinar a distintos tipos de pacientes, que realizan la "despedida" por motivos muy diferentes. En este caso intervienen otras variables, entre las que los tratamientos previos y quizás el consumo de otras drogas parecen tener la mayor importancia. Seguramente la "despedida" adquiere un significado inespecífico y distinto en cada enfermo, en función de las expectativas futuras debidas a los conocimientos y experiencias previos de tipo terapéutico. Quizás en unos es indicativo del final de una etapa y de la motivación para dejar de beber, mientras en otros sea por el contrario una expresión de resistencia.

El hecho de que la "despedida" sea especialmente de mal pronóstico entre los enfermos que ya habían realizado tratamientos previos respalda esta idea de que la motivación es distinta. Estos casos, por su experiencia anterior, es posible que piensen que siempre tienen la posibilidad de nuevos ingresos, mientras que en algunos pacientes "primerizos" la "despedida" puede ser reflejo de la ansiedad ante una situación desconocida de ingreso.

- También debemos tener en cuenta que la motivación y las fases del cambio no pueden plantearse por igual en pacientes que tienen un funcionamiento cognitivo distinto y en muchas ocasiones seriamente distorsionado. Precisamente entre los alcohólicos que ingresan abundan los casos de deterioro neuropsicológico, lo que, entre otras cosas, afecta a la capacidad de comprensión y aceptación de su enfermedad y a la capacidad para establecer estrategias conductuales de futuro y mantenerlas en el tiempo ${ }^{6}$. El hecho de que la conciencia de dependencia sea un factor determinante en el pronóstico de estos enfermos ${ }^{7}$ nos induce a pensar que la "despedida", en caso de deterioro cognitivo, puede reflejar, más que una falta de motivación, simplemente una severa incapacidad de autocontrol de la conducta.

- Finalmente debemos considerar el hecho mismo del ingreso y los objetivos terapéuticos que tiene. Entre estos se encuentra el lograr cambios significativos en la motivación, comprensión y conciencia de la dependencia alcohólica, con una disposición a modificar hábitos y conductas y una internalización del lugar de control de éstas conductas. Es probable que los terapeutas se adapten espontáneamente al estado motivacional de los pacientes, incidiendo de diferente manera en función de la fase en que se encuentran. El éxito del abordaje motivacional durante el ingreso de los pacientes menos motivados (los que tienen alcoholúrias positivas) se reflejaría precisamente en la difuminación de las diferencias previas al ingreso, entre las que puede haber la poca motivación a dejar de beber, expresada a través de la "despedida".

En conclusión, la "despedida" por si misma no parece ayudar a predecir la conducta futura en los enfermos alcohólicos que ingresan para desintoxicación, ya que puede obedecer tanto a una decisión de cambio personal ante el ingreso o a una resistencia hasta el último momento, pero combinada con otras conductas y características del paciente, especialmente los antecedentes de tratamientos y el estado neuropsicológico, puede darnos pistas acerca de sus actitudes respecto al futuro.

Comparado con los pacientes ambulatorios, en los que sí hay relación entre últimos consumos y abstinencia posterior ${ }^{9}$, hay que tener en cuenta que la situación de ingreso es por sí misma un cambio brusco y artificial en la conducta y también que los que precisan de él representan un subgrupo de entre los alcohólicos, con características diferenciales.

\section{REFERENCIAS}

1. Miller WR, Rollnick S (Eds.) Motivational Interviewing: Preparing People to Change Addictive Behavior. NY: Guilford Press; 1991.

2. Prochaska JO, Diclemente CC. Transtheoretical therapy: Toward a more integrative model of change. Psychother Theory Res Pract 1982; 19: 276-288.

3. Prochaska JO, Diclemente CC. The Transtheoretical approach: Crossing the traditional boundaries of therapy. Homewood, IL: Dorsey Press; 1984.

4. Ries RK, Dyck DG, Short R, Srebnik D, Snowden M, Comtois KA. Use of case manager ratings and weekly urine toxicology tests among outpatients with dual diagnoses. Psychiatr Serv. 2002; 53: 764-6.

5. Sharpe PC. Biochemical detection and monitoring of alcohol abuse and abstinence. Ann Clin Biochem. 2001; 38 (Pt 6): 652-664.

6. Monras M, Freixa N, Lligoña A, Gual A. Defensividad psicológica y deterioro cognitivo en alcohólicos. ¿Están relacionados y son útiles para el pronóstico a los 5 años?. Psiquiatría Biológica 1999; 6: 154-168.

7. Monras M, Garcia S, Torres M, Gual A, Ortega L. El ingreso hospitalario en el tratamiento de los alcohólicos cronificados. Adicciones 1998; 10: 151-160.

8. Monras M, Gual A, Freixa N. Situaciones especiales de la terapia grupal para alcohólicos: el paciente reacio a iniciar tratamiento Adicciones 1998; 10: 33-41.

9. Gual A, Lligoña A, Colom J. Five-year outcome in alcohol dependence. A naturalistic study of 850 patients in Catalonia. Alcohol Alcohol (England) 1999; 34: 183-192. 
